\title{
Comparação do gasto calórico e da frequência cardíaca em mulheres nas aulas de zumba presencial e virtual
}

\author{
Comparison of caloric expenditure and heart frequency in women in the presence and virtual \\ zumba classes
}

Comparación del gasto calórico y la frecuencia cardíaca en mujeres en aulas y clases virtuales de zumba

Recebido: 22/09/2021 | Revisado: 30/09/2021 | Aceito: 04/10/2021 | Publicado: 05/10/2021

\author{
Sheylla Araújo de Melo \\ ORCID: https://orcid.org/0000-0002-3814-067X \\ UNIESP Centro Universitário, Brasil \\ E-mail: sheyllaaraujom@gmail.com \\ Luís Filipe Gomes Barbosa Pereira de Lemos \\ ORCID: https://orcid.org/0000-0001-8431-3642 \\ Universidade Federal da Paraíba, Brasil \\ E-mail: luis.training75@gmail.com \\ Géssika Araújo de Melo \\ ORCID: https://orcid.org/0000-0003-2938-7280 \\ Universidade Federal da Paraíba, Brasil \\ E-mail: gessika.fisio@gmail.com
}

\begin{abstract}
Resumo
O presente estudo comparou o gasto calórico e a variação em mulheres nas aulas de zumba presencial e virtual. Tratou-se de uma pesquisa exploratória, descritiva e transversal, do tipo quantitativo. A amostra foi composta por dez mulheres, com média de idade de $36( \pm 4,7)$ anos, que participaram de duas aulas de zumba, sendo uma presencial e uma virtual. Os instrumentos utilizados para a coleta foram um questionário sociodemográfico, um frequencímetro, uma televisão e um Xbox 360. Os dados coletados foram analisados através do programa estatístico SPSS 24.0. Os resultados demonstraram que não houve diferença estatisticamente significativa em relação ao gasto calórico, quando comparadas as duas modalidades de aulas de zumba $(\mathrm{p}=0,221)$. Porém, observou-se uma diferença estatisticamente significativa em relação à frequência cardíaca média, sendo a aula presencial a que obteve maior índice $(\mathrm{p}=0,005)$. Portanto, ao comparar os dois modos de aulas de zumba, presencial e virtual, o estudo revelou que não houve diferença em relação ao gasto calórico nestas aulas. Entretanto, a intensidade da aula presencial permaneceu de maneira mais intensa e uniforme, comprovados pela superioridade nos valores da FCmed, na aula presencial.
\end{abstract}

Palavras-chave: Dança; Gasto energético; Jogos de vídeo.

\begin{abstract}
The present study compared caloric expenditure and variation in women in face-to-face and virtual zumba classes. It was an exploratory, descriptive and cross-sectional, quantitative study. The sample consisted of ten women, with an average age of $36( \pm 4.7)$ years, who participated in two zumba classes, one in person and one virtual. The instruments used for the collection were a sociodemographic questionnaire, a frequency meter, a television and an Xbox 360 . The data collected were analyzed using the statistical program SPSS 24.0. The results showed that there was no statistically significant difference in relation to caloric expenditure, when comparing the two types of zumba classes $(\mathrm{p}=0.221)$. However, there was a statistically significant difference in relation to the average heart rate, with the classroom class having the highest index $(\mathrm{p}=0.005)$. Therefore, when comparing the two modes of zumba classes, face-to-face and virtual, the study revealed that there was no difference in terms of caloric expenditure in these classes. However, the intensity of the face-to-face class remained more intense and uniform, evidenced by the superiority in the values of FCmed, in the face-to-face class.
\end{abstract}

Keywords: Dance; Energy expenditure; Video games.

\section{Resumen}

El presente estudio comparó el gasto calórico y la variación en mujeres en aulas y clases virtuales de zumba. Fue un estudio exploratorio, descriptivo y transversal, cuantitativo. La muestra consistió en diez mujeres, con una edad promedio de $36( \pm 4.7)$ años, que participaron en dos clases de zumba, una en persona y una virtual. Los instrumentos utilizados para la recopilación fueron un cuestionario sociodemográfico, un medidor de frecuencia, un televisor y una Xbox 360. Los datos recopilados se analizaron utilizando el programa estadístico SPSS 24.0. Los resultados 
mostraron que no hubo diferencias estadísticamente significativas en relación con el gasto calórico, al comparar los dos tipos de clases de zumba $(\mathrm{p}=0.221)$. Sin embargo, hubo una diferencia estadísticamente significativa en relación con la frecuencia cardíaca promedio, con la clase en el aula con el índice más alto $(\mathrm{p}=0,005)$. Por lo tanto, al comparar los dos modos de clases de zumba, cara a cara y virtual, el estudio reveló que no había diferencia en términos de gasto calórico en estas clases. Sin embargo, la intensidad de la clase cara a cara se mantuvo más intensa y uniforme, evidenciada por la superioridad en los valores de FCmed, en la clase cara a cara.

Palabras clave: Danza; Gasto de energía; Video juegos.

\section{Introdução}

A zumba foi criada em 1990, a partir da mescla de passos de dança e ginástica, proporcionando um sistema dinâmico que combina ritmos de diversas velocidades, permitindo aos participantes acompanhar facilmente os movimentos. A aula de zumba promove aos praticantes um bem-estar físico e mental por ser planejada com passos simples, música motivacional e uma metodologia gestual. Inspira-se em ritmos latinos e música internacional e executa-se de forma intermitente entre músicas de velocidades lenta e rápida. Isto facilita a integração de alguns dos princípios básicos do treinamento aeróbico, como o treinamento intervalado e treino de resistência, proporcionando maior gasto calórico e tonificação corporal (Zumba Fitness LLC, 2014). Luettgen (2012) destaca que, embora a Zumba seja amplamente conhecida, há poucas pesquisas analisando os potenciais benefícios dessa forma de dança para o corpo.

Com o avanço tecnológico surgiram alternativas diferenciadas de exercício físico, associando a facilidade de treino à praticidade, devido à possibilidade de sua realização em domicílio. Esse avanço trouxe o surgimento de jogos como os exergames, com os quais o indivíduo pode jogar de maneira dinâmica e divertida, movendo todo o corpo, sem a necessidade de utilizar controle remoto.

De acordo com o manual da Zumba Fitness® (2017), os jogos ativos de zumba, conhecidos como exergames ou zumba games permitem a execução do programa em qualquer lugar. Esses jogos são muito utilizados nos consoles do modelo Xbox 360, que correspondem a microcomputadores desenvolvidos para tal finalidade. Nesta atividade, o participante se posiciona em frente ao aparelho de vídeo game e, após ser localizado pelo sensor, inicia sua atividade de forma auto assistida, devendo apenas seguir os passos apresentados na tela.

Os jogos ativos promovem adaptações no nível de aptidão física (Perrier-Melo et al., 2016), aumento no gasto energético (Bailey \& Mcinnis, 2011) e elevam favoravelmente a frequência cardíaca (FC) em relação ao repouso (Canabrava, 2013). Assim, considerando o aumento da população que busca mudança de estilo de vida através da prática de exercício físico, a grande procura pelas aulas de zumba (dança/ginástica), e considerando ainda a possibilidade de utilização dos jogos ativos para o mesmo fim, houve o interesse em investigar a quantidade de gasto calórico resultante de uma aula de zumba no modo presencial e no modo virtual, pois ainda são escassos os estudos acerca da temática.

Diante do exposto, o presente estudo objetivou comparar o gasto calórico em mulheres nas aulas de zumba presencial e virtual. Para tanto, buscou-se verificar o gasto calórico em mulheres durante essas aulas, com o intuito de verificar se a aula de zumba por meio da realidade virtual pode ser considerada um meio efetivo de realização de exercício físico. Secundariamente, buscou-se investigar a diferença entre as $\mathrm{FC}_{\text {méd }}$ entre ambas as modalidades de aulas de zumba, com o intuito de observar em qual destas houve manutenção da intensidade de aula suficiente para promover respostas cardiovasculares agudas. Desta forma, a hipótese experimental foi de que "haveria diferença estatisticamente significativa no gasto energético entre os grupos de aula de zumba presencial e virtual".

\section{Metodologia}

Trata-se de um estudo quase experimental, descritivo, com abordagem quantitativa (Pereira et al., 2018). A amostra foi por conveniência, de caráter não probabilístico. Concluíram o protocolo 10 participantes do sexo feminino, com idades 
entre 29 a 46 anos, praticantes de zumba fitness em uma academia de ginástica da cidade de João Pessoa na Paraíba, que concordaram em participar da investigação.

Seguiram-se os critérios de inclusão: ser do sexo feminino; estar participando das aulas de zumba regularmente há, no mínimo, oito meses; ter idade entre 28 e 46 anos; e assinar o Termo de Consentimento Livre e Esclarecido (TCLE). Foram adotados como critérios de exclusão: indivíduos que desistiram da pesquisa; os que não compareceram no dia da coleta; apresentaram qualquer outra intercorrência no dia do estudo ou que declararam ter doenças respiratórias que dificultassem a realização do protocolo do estudo. As participantes foram orientadas a estarem pelo menos $24 \mathrm{~h}$ sem praticarem exercícios. Além disso, foram informadas que só poderiam entrar em repouso total após o término da aula.

Inicialmente, aplicou-se o questionário sociodemográfico para caracterização da amostra pesquisada. Em seguida, foram formados dois grupos organizados aleatoriamente através da permuta em blocos na taxa de 1:1, utilizando-se o programa de randomização online (www.random.org), com a finalidade de compor dois grupos com número de participantes equivalentes. Estes grupos foram formados com o intuito de que cada um deles iniciasse a coleta por uma modalidade de aula de zumba (presencial ou virtual) e, assim, minimizar o efeito de aprendizagem, visto que em ambas as aulas foram utilizadas a mesma sequência coreográfica. As participantes não tinham experiências com o Xbox 360 e o jogo zumba fitness core.

A aparelhagem utilizada foi uma televisão de 32" com um suporte estático; um console Xbox 360; um Kinect ${ }^{\mathrm{TM}}$, sensor que permite a leitura dos movimentos do jogador através de duas câmeras, eliminando a necessidade do controle remoto; um jogo Zumba Fitness Core ${ }^{\circledR}$ e um monitor de frequência cardíaca de marca Polar FT7. Este último foi utilizado para verificar o gasto calórico e a frequência cardíaca e corresponde a um aparelho composto por um relógio de pulso e uma cinta elástica que é usada em contato com a pele, na altura do esterno.

O primeiro grupo (G1) foi formado por cinco mulheres que iniciaram a coleta pelas aulas de zumba presencial. A coleta foi realizada com a presença ativa do instrutor, que se posicionou frontalmente à turma e orientou os movimentos coreográficos através de gestos com os braços e as mãos. Cada participante foi orientada a reproduzir os movimentos do instrutor, que estava repassando ao grupo as coreografias do jogo aprendidas anteriormente, durante os 45 minutos de aula.

O segundo grupo (G2) foi formado por cinco mulheres que iniciaram a coleta pelas aulas virtuais de zumba e essas deveriam seguir os movimentos apresentados no vídeo. Nesta modalidade, a indicação dos movimentos é feita através de uma janela, localizada no canto superior direito da tela, na qual aparecem os próximos movimento alguns segundos antes da mudança. Cada participante foi orientada a reproduzir os movimentos do jogo durante os 45 minutos de aula virtual.

Ambas as aulas foram realizadas duas a duas, de maneira que a quinta participante realizou a aula em companhia de uma sexta mulher, a qual não era participante da pesquisa e, portanto, não fez uso do aparelho frequencímetro. Ao término da primeira fase, o G1 passou a fazer as aulas no modo virtual e o G2 no modo presencial. Foram utilizadas as mesmas músicas e coreografias para os dois estilos de aula, sendo ambos realizados sempre no mesmo turno.

Quanto aos aspectos éticos, o presente estudo teve início após aprovação do Comitê de Ética em Pesquisa do Centro Universitário Maurício de Nassau - UNINASSAU, sob o número da Plataforma Brasil 2.326.597. Todos os sujeitos assinaram o Termo de Consentimento Livre e Esclarecido - (TCLE). O estudo seguiu as normas da Resolução n ${ }^{\circ} 466$ de 12 de dezembro de 2012 do Conselho Nacional de Saúde, em vigor no país, que regulamenta pesquisa envolvendo seres humanos.

Utilizou-se o software SPSS, versão 24, para análise dos dados de gasto calórico e frequências cardíacas média e máxima. A comparação dos dados entre as aulas presencial e virtual foi realizada através do teste não paramétrico de medidas pareadas, o teste de Wilcoxon. O nível de significância foi estabelecido em $\mathrm{p}<0,05$. 


\section{Resultados}

Os dados sociodemográficos relacionados ao estado civil, escolaridade e renda estão na tabela 1.

Tabela 1. Dados sociodemográficos dos participantes da pesquisa.

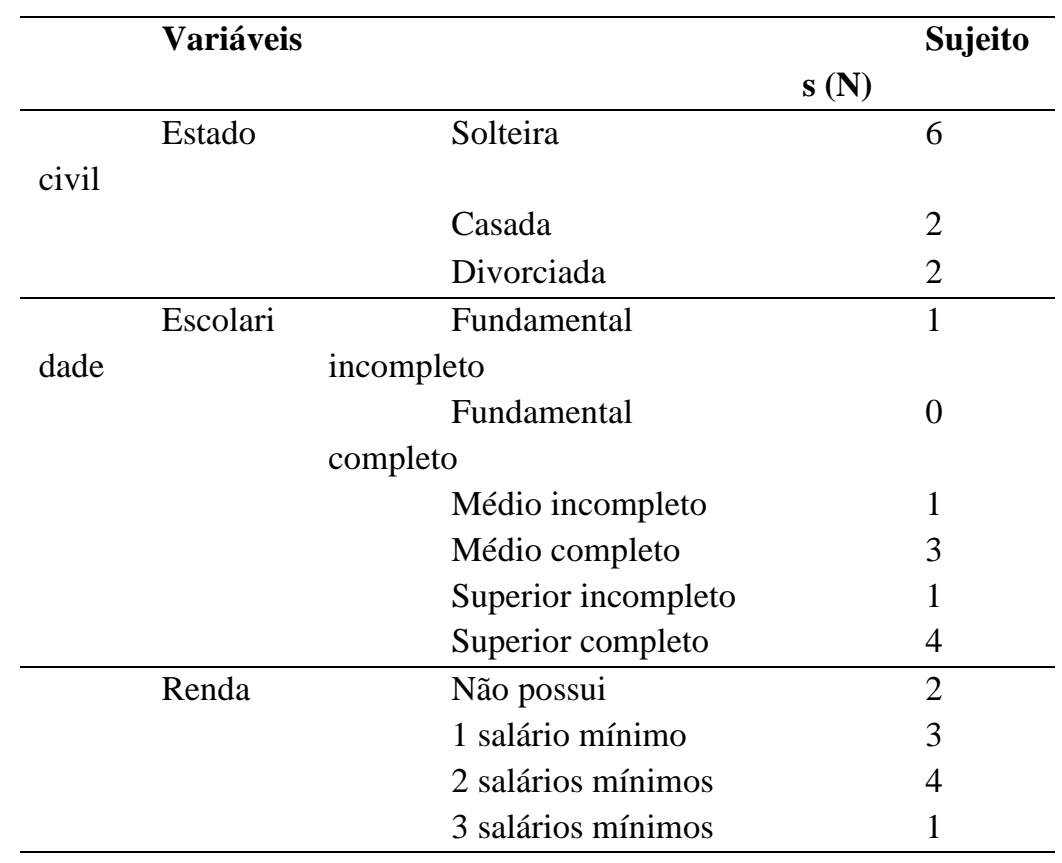

Fonte: Dados da pesquisa (2017).

Assim, a maioria era solteira (60\%), com nível superior completo (40\%) e com renda de dois salários mínimos (40\%). A média de idade da amostra foi de $36(\mathrm{DP}= \pm 4,7)$ anos, com peso médio de 70,2 $(\mathrm{DP}= \pm 12,0)$ quilos e altura média de 159 $(\mathrm{DP}= \pm 8,4)$ centímetros. Os dados descritivos relacionados ao gasto calórico, Frequência Cardíaca Máxima $\left(\mathrm{FC}_{\mathrm{Max}}\right)$ e Frequência Cardíaca Média ( $\left.\mathrm{FC}_{\text {méd }}\right)$ nas aulas de zumba presencial e virtual estão expostos na tabela 2.

Assim, percebe-se que o gasto calórico médio na aula presencial foi de $393(\mathrm{DP}= \pm 88,3)$ kcal e na aula virtual foi de 356,1 (DP $= \pm 105,2$ ), mostrando uma leve superioridade para o primeiro tipo de aula de zumba (presencial). Os dados referentes às frequências cardíacas demonstram valores muito próximos de $\mathrm{FC}_{\text {máx }}$ e valores mais distintos de $\mathrm{FC}_{\text {méd }}$ entre os dois tipos de aula (Tabela 2).

Tabela 2. Dados descritivos dos participantes da pesquisa.

\begin{tabular}{l|c|c}
\hline Variáveis & Zumba presencial & Zumba virtual \\
\hline Gasto Calórico (kcal) & $393,0( \pm 88,3)$ & $356,1( \pm 105,3)$ \\
FC Média $(b p m)$ & $150,3( \pm 16,9)$ & $143,2( \pm 16,3)$ \\
FC $_{\text {Máxima }}(\mathrm{bpm})$ & $174,8( \pm 13,3)$ & $176,5( \pm 16,7)$ \\
\hline
\end{tabular}

Fonte: Dados da pesquisa (2017).

Percebe-se que, em relação às médias, houve maior gasto energético e $\mathrm{FC}_{\text {méd }}$ na aula presencial de zumba presencial, enquanto que a $\mathrm{FC}_{\text {máx }}$ foi superior na aula virtual. Em relação às estatísticas inferenciais, o teste de Wilcoxon para amostras relacionadas mostrou que não houve diferença estatisticamente significativa entre o gasto calórico nas aulas de zumba presencial e virtual $(\mathrm{p}=0,221)$. Portanto, ambos os tipos de aula de zumba, apresentaram valores de gasto calórico semelhante e se pode inferir que proporcionam ao corpo o mesmo consumo energético. 
No tangente à comparação da $\mathrm{FC}_{\text {méd }}$ os dados foram estatisticamente diferentes nas duas aulas ( $\left.\mathrm{p}=0,005\right)$, na qual a presencial mostrou maiores níveis de $\mathrm{FC}_{\text {méd }}$ quando comparada com a virtual. Para a $\mathrm{FC}_{\text {máx }}$, os resultados apontam para uma igualdade entre os valores, pois $\mathrm{p}=0,766$, indicando que não houve diferença estatisticamente significativa entre os achados. Logo, ambos os tipos de aulas de zumba proporcionam níveis de $\mathrm{FC}_{\text {máx }}$ semelhantes.

\section{Discussão}

O desenvolvimento de desordens relacionadas à saúde, tais como obesidade, diabetes ou doença cardiovascular estão diretamente relacionadas ao sedentarismo (Lee et al., 2012). Por isso, é de suma importância favorecer o interesse e a participação no exercício físico para melhorar a saúde e prevenir a obesidade (Delextrat \& Neupert, 2015).

Segundo Neves et al. (2015), o entendimento das respostas de gasto calórico e cardiovasculares ao exercício físico é crucial para a obtenção de valores que permitam adequar os programas de treinamento e garantir a segurança dos profissionais. Desta forma, tem-se investigado acerca da capacidade de influenciar o gasto calórico e respostas cardiovasculares agudas durante aulas de zumba fitness presencial e em ambiente de realidade virtual (Neves et al., 2015). Sabe-se que as aulas de zumba integram alguns princípios do treino aeróbio com intervalo e treino de resistência, tonificando o corpo, promovendo benefícios cardiovasculares e aumentando a queima calórica, associando o treino aeróbio com o treinamento intermitente (Zumba Fitness LLC, 2014).

No presente estudo, ao comparar o gasto calórico em mulheres nas aulas de zumba presencial e virtual com a utilização do console Xbox 360, observou-se que não houve diferença estatisticamente significativa. De acordo com o American College of Sports Medicine (ACSM), o indicado é que os indivíduos gastem $300 \mathrm{kcals}$ por treino para que possa haver perda de peso e manutenção de um peso corporal saudável (ACSM, 2010). Portanto, ambas as formas de prática da Zumba foram efetivas em relação ao gasto calórico.

Em relação à resposta fisiológica na frequência cardíaca média nas aulas presenciais, percebeu-se uma diferença estatisticamente significativa. A partir deste achado, pode-se inferir que a aula de zumba presencial manteve sua intensidade geral, no decorrer da aula, por um período mais prolongado e de maneira mais homogênea. Nesse contexto, destaca-se que a presença de outras pessoas interfere na motivação das mulheres para se esforçarem durante a execução do exercício (Wininger \& Pargman, 2003), o que pode justificar a superioridade do grupo presencial em relação a esta variável. No estudo de Ferreira (2014), realizou-se uma caracterização de intensidade de esforço de uma aula de zumba com mulheres entre 18 e 64 anos. Assim, observou que, independentemente do nível de aptidão física, todas as mulheres alcançaram valores de $\mathrm{FC}_{\operatorname{máx}}$, reforçando os dados que apontam que a zumba é um bom exercício para se alcançar respostas cardiovasculares agudas. Em outro estudo, foi possível observar que em um grupo de 19 mulheres saudáveis praticantes de zumba, obteve-se a $\mathrm{FC}_{\text {méd }}$ em torno de 154 bpm (Luettgen et al., 2012), assemelhando-se ao presente estudo.

Outras pesquisas também encontraram aumento na FC após a realização de Zumba Fitness (Neves et al., 2015; Delextrat \& Neupert, 2015). Este fato se justifica pelos aspectos psicológicos e sociais envolvidos na prática de exercício físico. Segundo Delextrat e Neupert (2015), o exercício físico realizado em grupo ou sozinho apresenta diferenças. A presença do professor de dança, instruindo as coreografias influencia as características psicológicas dos participantes, como em relação à motivação.

Além dos achados acima, outros estudos também apontaram aumento significativo da FC a partir da realização de aulas de Zumba Fitness em ambiente virtual, o qual se contrapõe aos resultados do presente artigo (Neves et al., 2015). Em seu estudo, Neves et al. (2015) apontam que nove homens e nove mulheres realizaram movimentos coreografados por 22 minutos utilizando o Xbox 360 Kinect, com jogo zumba fitness core, e observaram um aumento significativo de 67\% na FC.

Embora se tenha observado maior variação na FC no grupo de Zumba presencial, destaca-se que foi possível alcançar 
a média de 143,2 (DP = $\pm 16,3)$ bpm em relação à FC média. Este aspecto pode ser justificado pelo fato de que a atividade física em um ambiente virtual através do Xbox 360 pode alterar as respostas cardiovasculares agudas. Assim, apoia-se a viabilidade do uso da Zumba Fitness virtual através do Kinect para o Xbox 360 em programas de atividade física, com o intuito de favorecer respostas cardiovasculares em curto tempo (Neves et al., 2015), associada à prática regular deste exercício de maneira presencial, devido à necessidade de um acompanhamento profissional adequado.

No tocante ao gasto calórico, principal enfoque da presente pesquisa, verificou-se que não houve diferença estatisticamente significativa entre os grupos de zumbas virtual e presencial ( $p>0,05)$. Desta maneira, ambas as categorias de aulas promoveram gasto calórico semelhante, com discreta superioridade para o gasto na aula presencial.

Para diminuir o peso corporal, ou mantê-lo em níveis saudáveis, e melhorar a aptidão cardiovascular, preconizam-se gastos pelo menos $300 \mathrm{kcal}$ por exercício físico (Delextrat \& Neupert, 2015). Os resultados do presente estudo demonstraram a capacidade de ambas as aulas de zumba promoverem treino eficaz, de forma a alcançar os benefícios supracitados, visto que em ambas as aulas foi possível superar esses valores estimados.

O gasto de energia varia de acordo com o tipo de exercício e sua intensidade, assim como de indivíduo para indivíduo (Denadei \& Greco, 2011). Embora não tenha havido diferenças estatisticamente significativas entre os grupos, é inegável a capacidade destas modalidades de aulas de zumba proporcionarem um elevado gasto energético, ou seja, superior às 300 kcal preconizadas (Delextrat; Neupert, 2015).

Sabe-se da preponderância da zumba presencial em relação à zumba através do DVD ou videogame. No experimento de Delextrat e Neupert (2015), comparou-se uma aula de zumba presencial com uma aula com DVD, realizada por quinze mulheres com idade média de 25 anos. Ao final de seu estudo, pôde ser constatado que as aulas presenciais tiveram um maior gasto energético que as realizadas com um DVD. Este resultado é similar aos achados da presente pesquisa que, embora a comparação dos valores referentes ao gasto calórico não tenha sido diferente estatisticamente, foi possível observar que o gasto calórico foi superior na aula de zumba presencial.

Entretanto, também se sabe que o uso de games promove gasto calórico e aumento da frequência cardíaca durante o exercício, configurando-se como importante meio complementar para a promoção da saúde e recurso contra a obesidade. Outros benefícios encontrados nos estudos com exergames são a melhora do equilíbrio, o controle postural e a reabilitação dos segmentos corporais (Vaghetti \& Botelho, 2010).

Outros autores revelam que os jogos ativos, muito utilizados nos consoles Xbox 360, promovem aumento na flexibilidade, melhora na resistência muscular localizada, desenvolvem e mantêm o nível de aptidão física em adultos não treinados (Perrier-Melo et al., 2016). Os movimentos corporais realizados em função dos exergames desencadeiam várias adaptações fisiológicas, como o aumento no gasto energético, na intensidade da atividade (Bailey \& Mcinnis, 2011; Graves et al., 2010) e frequência cardíaca (Graves et al., 2010).

Bailey e Mcinnis (2011) observaram em seu estudo que a utilização de jogos para promover o exercício físico eleva o gasto de calórico para intensidade moderada ou vigorosa, concluindo que os exergames são capazes de aumentar a intensidade da atividade física e favorecer o equilíbrio energético. Além disso, Melo et al. (2017) e Pierre-Melo et al. (2016) afirmam que o ambiente virtual por meio do console Xbox 360, promove uma melhora no equilíbrio de pacientes com hemiparesia, aumento na flexibilidade, melhora na resistência muscular, desenvolve e mantém o nível de aptidão física em adultos saudáveis não treinados.

Como o movimento humano é uma característica fundamental dos exergames, esse jogo se tornou uma nova ferramenta para as ciências da saúde, principalmente para educação física. Estudos apontaram que os exergames são muito utilizados por profissionais de educação física e fisioterapeutas nas aulas e em trabalho com reabilitação. Nas aulas de educação física, algumas escolas dos Estados Unidos utilizam esses jogos ativos por proporcionarem significativo gasto 
calórico, associado ao lúdico entretenimento (Vaghetti \& Botelho, 2010).

Segundo Meneghin et al. (2016), os exergames têm se destacado no cenário da prática de exercício físico, pois possibilitam ao indivíduo autonomia quanto aos dias e horários de prática, além do tipo de jogo e intensidade desejada. Assim, os jogos ativos podem ser praticados em diversos locais e o praticante pode optar por jogar sozinho, em dupla ou em grupo, favorecendo, por vezes, a motivação à prática deste exercício.

Diante deste cenário, estes jogos se tornaram uma atividade presente em todas as partes da sociedade atual e os usuários os utilizam como meio de diversão que possibilita efetivo gasto calórico. Posto isto, Corrêa et al. (2011), relatam que nos últimos tempos foi crescente a procura por esse tipo de tecnologia como um meio de incentivo inicial à prática de exercício físico.

Em decorrência da escassez de literatura a respeito da quantificação do gasto energético em aulas de zumba presencial e virtual utilizando o Xbox 360, é possível observar a valiosa contribuição do presente trabalho neste cenário. Assim, enfatizou-se uma reflexão acerca desta temática, reforçando ambos os tipos de aula de zumba como eficazes para promover o gasto calórico necessário para obtenção de benefícios fisiológicos aos praticantes. Assim, a zumba através do Xbox 360 se configura como uma alternativa complementar à zumba presencial, pois é capaz de promover gasto calórico semelhante a esta modalidade de atividade física.

\section{Conclusão}

Ao comparar os dois modos de aulas de zumba, presencial e virtual, o estudo revelou que não houve diferença em relação ao gasto calórico nestas aulas. Entretanto, a intensidade da aula presencial permaneceu de maneira mais intensa e uniforme, comprovados pela superioridade nos valores da $\mathrm{FC}_{\text {méd, }}$, na aula presencial.

Por promover um gasto calórico semelhante ao da aula de zumba presencial e por este gasto ser superior ao preconizado na literatura para promover benefícios fisiológicos aos praticantes, sugere-se a utilização da zumba virtual como ferramenta complementar na realização de exercícios físicos domiciliar. Portanto, conclui-se, que ambos os tipos de aula de zumba são eficazes para promover o gasto calórico preconizado para obtenção de benefícios fisiológicos necessários ao bom exercício físico.

Algumas limitações foram identificadas no presente estudo, como o número amostral reduzido e a ausência da avaliação dos aspectos motivacionais e psicológicos nas duas modalidades de aulas de zumba. Segundo Ortís et al. (2007), as características psicológicas dos praticantes das aulas de dança podem influenciar as respostas fisiológicas de exercícios. Assim, a avaliação dos aspectos psicológicos poderia auxiliar na elucidação da relação entre gasto calórico e motivação com as aulas de zumba. Sugerem-se, portanto, posteriores abordagens desta temática, a fim de possibilitar maior aprofundamento acerca deste campo do saber.

\section{Referências}

American College of Sports Medicine. (2013). ACSM's guidelines for exercise testing and prescription. Lippincott Williams \& Wilkins.

Bailey, B. W., \& McInnis, K. (2011). Energy cost of exergaming: a comparison of the energy cost of 6 forms of exergaming. Archives of Pediatrics \& Adolescent Medicine, 165(7), 597-602.

Canabrava, K. (2013). Gasto energético e intensidade das atividades físicas dos jogos ativos de vídeo games em crianças e adolescentes, (Tese de doutorado). Viçosa: Universidade Federal de Viçosa.

Corrêa, A. G. D., Monteiro, C. B. D. M., Silva, T. D. D., Lima-Alvarez, C. D. D., Ficheman, I. K., Tudella, E., \& Lopes, R. D. D. (2011). Realidade virtual e jogos eletrônicos: uma proposta para deficientes. Realidade virtual na paralisia cerebral. São Paulo: Plêiade.

Delextrat, A, \& Neupert, E. (2016). Physiological load associated with a Zumba® fitness workout: a comparison pilot study between classes and a DVD. Journal of Sports Sciences, 34(1), 47-55. 
Denadai, B. S, \& Greco, C. C. (2005). Prescrição do treinamento aeróbio: teoria e prática. Guanabara Koogan.

Ferreira, J. S. V. (2014). Caracterização da Intensidade de Esforço de uma Aula de Zumba Fitness, (Dissertação Mestrado). Porto: Faculdade de Desporto da Universidade do Porto.

Graves, L. E., Ridgers, N. D., Williams, K, Stratton, G, Atkinson, G, \& Cable, N. T. (2010). The physiological cost and enjoyment of Wii Fit in adolescents, young adults, and older adults. Journal of Physical Activity and Health, 7(3), 393-401.

Lee, I. M., Shiroma, E. J., Lobelo, F., Puska, P., Blair, S. N., Katzmarzyk, P. T., \& Lancet Physical Activity Series Working Group. (2012). Effect of physical inactivity on major non-communicable diseases worldwide: an analysis of burden of disease and life expectancy. The lancet, 380(9838), 219-229.

Luettgen, M, Foster, C, Doberstein, S, Mikat, R, \& Porcari, J. (2012). ZUMBA®: Is the "fitness-party" a good workout?. Journal of Sports Science \& medicine, 11(2), 357-358.

Melo, G. A, Lemos, M. T. M., Carvalho, S. M. C. R., \& Germano, C. D. F. M. (2017). A Realidade Virtual no Treino do Equilíbrio Corporal na Hemiparesia causada por Paralisia Cerebral. Adolescencia e Saude, 14(2), 176-182.

Meneghini, V., Barbosa, A. R., Mello, A. L. S. F. D., Bonetti, A., \& Guimarães, A. V. (2016). Percepção de adultos mais velhos quanto à participação em programa de exercício físico com exergames: estudo qualitativo. Ciência \& Saúde Coletiva, 21, 1033-1041.

Neves, L. E. D. S., Cerávolo, M. P. D. S., Silva, E, De Freitas, W. Z, Da Silva, F. F., Higino, W. P., \& De Souza, R. A. (2015). Cardiovascular effects of Zumba ${ }^{\circledR}$ performed in a virtual environment using XBOX Kinect. Journal of Physical Therapy Science, 27(9), 2863-2865.

Ortís, L. C., Maymí, J. N., Feliu, J. C., Vidal, J. M. L., Romero, E. P., Bassets, M. P., \& Brosa, J. V. (2007). Exercise motivation in university community members: A behavioural intervention. Psicothema, 19(2), 250-255.

Pereira A. S. et al. (2018). Metodologia da pesquisa científica. UFSM.

Perrier-Melo, R. J., Brito-Gomes, J. L., Garrido, N. D., Oliveira, S. F., Guimarães, F. J. D. S. P., \& Costa, M. D. C. (2016). Efeito do treinamento com videogames ativos nas dimensões morfológica e funcional: estudo clínico randomizado. Motricidade, 12(2), 70-79.

Vaghetti, C. A. O., \& da Costa Botelho, S. S. (2010). Ambientes virtuais de aprendizagem na educação física: uma revisão sobre a utilização de Exergames. Ciências \& Cognição, 15(1), 76-88.

Wininger, S. R., \& Pargman, D. (2003). Assessment of factors associated with exercise enjoyment. Journal of Music Therapy, 40(1), 57-73.

Zumba Fitness, L. L. C. (2018). Site brasileiro oficial Zumba Fitness. em http://www.zumba.com/pt-BR/about.

Zumba Fitness, L. L. C. (2014). Manual de treinamento para o instrutor: passos básicos nível 1. https://www.zumba.com/pt-BR/about 